\title{
IN SILICO STUDY OF GUANOSINE TRIPHOSPHATE (GTP) AND RIBAVIRIN DERIVATIVES AS THE INHIBITOR FOR NS5 METHYLTRANSFERASE OF DENGUE VIRUS
}

\author{
U. S. F. Tambunan ${ }^{1 *}$, A. A. Parikesit ${ }^{1}$, F. Wardani ${ }^{1}$, M. A. F. Nasution ${ }^{1}$ \\ and D. Kerami ${ }^{2}$ \\ ${ }^{1}$ Bioinformatics Research Group, Department of Chemistry, Faculty of Mathematics and Natural \\ Science, 16424, Depok, Indonesia \\ ${ }^{2}$ Mathematics Computational Group, Department of Mathematics, Faculty of Mathematics and \\ Natural Science, 16424, Depok, Indonesia \\ *E-mail: usman@ui.ac.id
}

\begin{abstract}
Dengue virus (DENV) infection has become a major health threat lately, endangering at least one-third of world's population and infecting 100 million people each year. Thus, these problems can be overcome by finding new solutions to control the DENV contagion level are needed, and one of them can be achieved by finding new drugs. Ribavirin and guanosine triphosphate (GTP) derivatives can be used as an antiviral drug design for this disease, especially in inhibition of enzymatic activity of the methyltransferase (Mtase) that involves in synthesizing DENV cap-RNA. In this study, we used the in the silico study through molecular docking simulation using AutoDock Vina 1.0 and MOE 2008.10 software. In addition, the toxicity and synthetic accessibility prediction were conducted as well. The results show the modified ligand derivatives of GTP and RTP, such as viramidine, xanthosine, and inosine, have favorable inhibition activities in the DENV NS5 Mtase in terms of the binding affinity and their interactions with the important residues of the DENV NS5 Mtase active site. Moreover, these ligands also have good drug properties as well. As the conclusion, the viramidine, xanthosine, and inosine derived ligands can be developed as a lead candidate as DENV NS5 MTase inhibitors for dengue fever therapy.

Keywords: Dengue virus, NS5 methyltransferase, inhibitor, GTP, ribavirin, molecular docking, AutoDock Vina

(C) RASĀYAN. All rights reserved
\end{abstract}

\section{INTRODUCTION}

Dengue fever, which is caused by dengue virus (DENV), still becomes one of the most common and deadly diseases in the world especially in tropical and subtropical continents such as Asia, Africa, and South America. ${ }^{1}$ Based on the latest data from the World Health Organization (WHO), there are an estimated 50100 million cases of dengue infection worldwide every year. ${ }^{2}$ The transmission of DENV is caused by mosquitos from Aedes genus such as Aedes albopictus and Aedes aegypti. ${ }^{3}$ To date, DENV has four serotypes that can cause dengue fever to human (DENV-1, DENV-2, DENV-3, DENV-4), based on the antibody response that generated on the body when the DENV infects. The fifth serotype, DENV-5, was found recently in 2013 at Sarawak, Malaysia. Although the lethality of this serotype has yet to be known. ${ }^{4-}$ ${ }^{6}$ DENV is a key member of Flavivirus genus within Flaviviridae family. The genome itself is singlestranded, positive-sense enveloped virus which encodes three structural proteins (capsid (C), membrane (M) protein, and envelope (E) protein) and seven nonstructural (NS) proteins (NS1, NS2A, NS2B, NS3, NS4, NS4B, and NS5). ${ }^{7,8}$ The NS5 protein is the largest protein of all nonstructural protein in DENV; it comprises of two domains based on their enzymatic activities; the methyltransferase (MTase) domain at its N-terminal and RNA-dependent RNA polymerase (RdRp) at its C-terminal. ${ }^{9,10}$ The enzymatic activities of this protein are important for the RNA synthesis that needed for replication process of DENV. ${ }^{8}$ As such, both NS5 Mtase and NS5 RdRp domains play an imperative role in the viral life cycle.

Rasayan J. Chem., 10(3), 910-921(2017)

http://dx.doi.org/10.7324/RJC.2017.1031613 
In this research, ribavirin triphosphate (RTP) has been used as the template for the inhibition of NS5 Mtase, as the recent study shows that RTP can compete with guanosine triphosphate (GTP) in the GTP binding site of NS5 Mtase. ${ }^{11}$ Ribavirin is a drug precursor (prodrug) that undergoes a similar process to the metabolism of purine nucleotides in RNA. Ribavirin interferes with the metabolism of RNA that required for virus replication. As such, this research would observe the interaction of the compounds ribavirin and GTP derivatives as inhibitors of four serotypes of DENV NS5 Mtase by molecular docking. ${ }^{12}$ Furthermore, the synthetic accessibility and toxicity prediction were conducted as well.

\section{EXPERIMENTAL}

\section{General procedure}

In this study, the molecular structure of ribavirin and GTP were drawn by using ChemSketch 12.0 software, while the NS5 Mtase sequence of the protein database was retrieved from National Center for Biotechnology Information (NCBI) website (http://www.ncbi.nlm.nih.gov). Later, the 3D structure of DENV NS5 Mtase virus was downloaded from the Research Collaboratory for Structural BioinformaticsProtein Data Bank (RCSB-PDB) database (http://www.rcsb.org/pdb/home/home.do). Grid box was a necessary parameter in the process of AutoDock ligand docking with target enzyme, the preparation itself was done by converting the file docking ligands to the .pdbqt file format. Docking simulation was performed by using AutoDock Vina 1.0 software with the adjusted parameters, based on our validated in silico approach ${ }^{13,14}$, while the analysis of the docking result was performed using MOE 2008.10 software and PyMol. In this step, we selected the best ligands from the docking simulation, based on the $\Delta \mathrm{G}_{\text {binding }}$ value and the interaction with NS5 Mtase, for drug scan analysis, including the toxicity and synthetic accessibility prediction. The drug scan analysis was done by comparing these ligands into the Lipinski's Rule of Five as well as their oral bioavailabilities. ${ }^{15}$ The toxicity analysis, including the carcinogenicity and mutagenicity prediction, was performed by using Lazar and Toxtree software (toxtree.sourceforge.net/). In order to help predict whether the wet laboratory could synthesize a particular design of molecules/ligands, Synthetic Accessibility (SA) parameter was used. In this study, we used CAESA (Computer Assisted Estimation of Synthetic Accessibility) to determine the SA of the best ligands. ${ }^{16}$

\section{Pre-docking Preparation}

\section{RESULTS AND DISCUSSION}

The structure of ribavirin, GTP, and their derivatives were designed by using the software ChemSketch 12.0. In this study, guanosine, ribavirin and their derivatives such as xanthosine, inosine (GTP derivatives) and viramidine (ribavirin derivatives) were used. In addition to these compounds, emtricitabine (GTP derivates) were also added as well. ${ }^{17}$ The ligand modifications were made at the cyclical chain functional groups and the ligand candidate compounds. As described in Fig.-1, the modified site was located at 2'O and $3^{\prime} \mathrm{O}$ groups on the ribose sugar and phosphate groups. ${ }^{18}$

The modification of phosphate group was done to fulfill one of the Lipinski's Rule of Five terms (Molecular weight, MW, less than 500 Daltons). ${ }^{19}$ In addition, through the modification, it would be seen whether the increase or decrease in the phosphate groups could affect the interaction of ligands with the important residues of NS5 Mtase. ${ }^{20}$ As a result, 510 ligand compounds were generated through this modification ${ }^{21}$, the example of the modified compounds is showed in Fig. -2 .

The DENV NS5 Mtase sequences that we used as the target enzyme was concluded by using NCBI database from its official website. ${ }^{22}$ In this point, we obtained ten DENV NS5 Mtase sequences from various countries. Afterward, all of the sequences were collected in order to perform multiple sequences alignment. These were done to find a sequence that can represent all existing NS5 Mtase sequence from the similarity score that was produced in the alignment process. The NS5 Mtase sequence which has the highest similarity score (maximum score of 100) will be selected. After that, the homology modeling of DENV NS5 Mtase would be done by first selected the chosen NS5 Mtase from the previous step in FASTA file format, then reproduce the 3D structures by using SWISS-MODEL. ${ }^{23,24}$ In this study, we used the PDB ID: 2P3O as the 3D model of our NS5 Mtase protein. ${ }^{25}$ 


\section{Docking Simulation}

A total of 510 ligands from our previous step was prepared for the molecular docking simulation. During the screening process, each molecule produces a series of positions through specific conformation and orientation of the enzyme-ligand complex. In this research, the docking simulations were done using MOE 2008.10 and AutoDock Vina software.

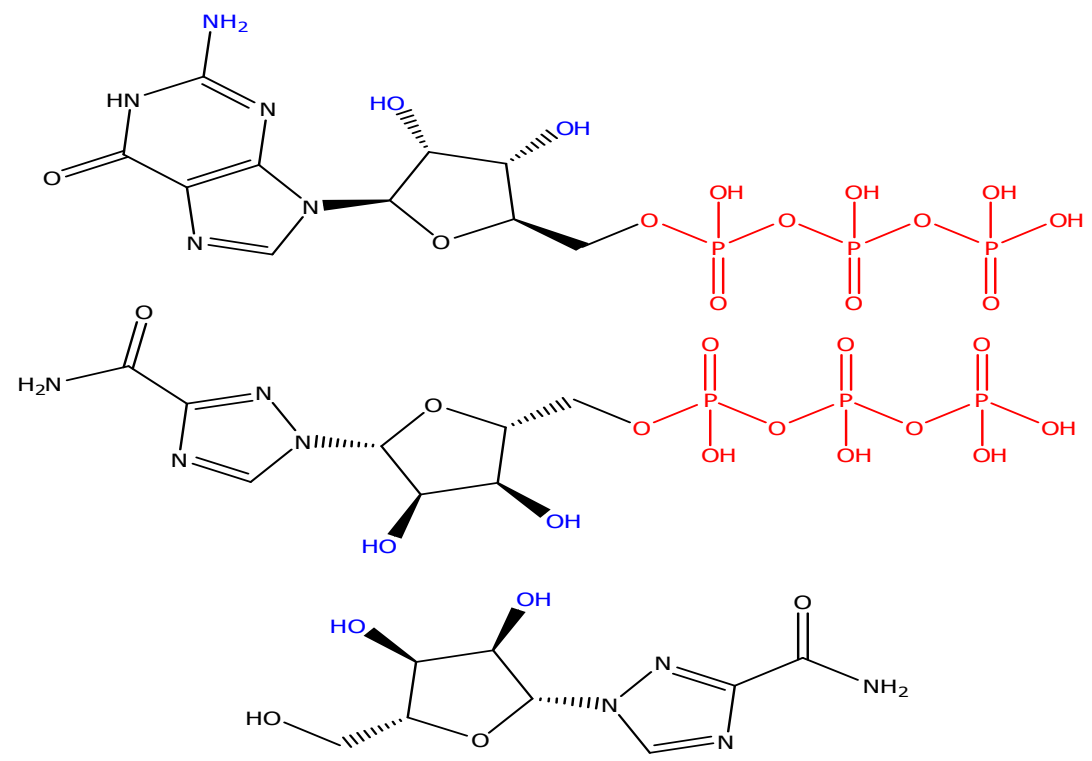

Fig.-1: The modification of the ligands in this study, namely GTP (top), RTP (middle), and ribavirin (bottom). The red fonts indicate that these functional groups were modified into either monophosphate (MP), diphosphate (DP), or triphosphate (TP), while the blue font indicates that the functional group modifications occurred on this site.<smiles>NC(=O)NC(=O)c1cn(C2OC(COP(=O)(O)OP(=O)(O)OP(=O)(O)O)C(N)C2O)cn1</smiles>

Fig.-2: Xanthosine_N3_TP_NS.

The screening process using MOE 2008.10 was aimed to see the $\Delta \mathrm{G}_{\text {binding }}$ value of each ligand to their receptor (NS5 Mtase). First, the 3D structure of DENV NS5 Mtase structure was optimized and minimized using 'Protonate 3D' and 'Energy Minimization' feature, respectively, on 'Compute' module in MOE 2008.10 software. This step was required to determine the three-dimensional coordinates of the hydrogen atoms in the NS5 Mtase since the PDB files do not include the hydrogen atoms in the enzyme structure. The hydrogen atoms are required for molecular mechanics, electrostatic calculation, and molecular docking. Furthermore, the DENV NS5 Mtase has also been protonated with 'Partial Charge' feature in MOE 2008.10 
RASĀYAN J. Chem.

Vol. 10 | No. 3 |910 - 921 | July - September | 2017

as well through the selected parameters and force fields. In this study, we used AMBER99 and MMFF94x as the force fields to protonate the DENV NS5 Mtase. The force fields were chosen because these force fields are suitable for the macromolecules, such as proteins and enzymes compared to the other force fields. Furthermore, these parameters were used in the previous study. ${ }^{26,27}$ After that, the minimization of DENV NS5 Mtase structure was performed to calculate the initial geometry and conformation of the enzyme. The same with the previous step, the energy minimization of this step was done by applying the same force fields: AMBER99 and MMFF94x with a gradient of $0.05 \mathrm{kcal} / \AA$.

All ligands which had previously been converted into MDL Mol format can be directly opened by MOE 2008.10 software to do further optimization through MOE Database Viewer. First, the ligands optimization step started by using 'Wash' protocol through standard parameters to improve the initial geometry and position of the hydrogen atoms of the ligands. Then, the optimization process continued by adding force field to the ligands using MMFF94x with RMS gradient of 0.01. The rest of parameters were adjusted using the default setting of the MOE 2008.10 software.

After the ligands and NS5 Mtase enzyme had been prepared, the next step of this phase was docking simulation by using 'Dock' feature on 'Compute' option of MOE 2008.10. In this study, we selected 'Triangle Matcher' parameter as the placement method, which is the default parameter of the software. This parameter showed the random movement of the ligand in the enzyme's active site to produce the most optimal ligand's orientation and position. As described by Podvinec et al. ${ }^{27}$, the active site of DENV NS5 Mtase lies on Lys14, Leu17, Asn18, Leu20, Lys22, Phe25, Lys29, Ser150 and Ser150 residues. Therefore, we selected these residues as the binding site of NS5 MTase through 'Site Finder' feature on MOE 2008.10. The initial screening results, using AMBER99 and MMFF94x force field, can be seen in Table-1 and Table2 , respectively.

Table-1: Ligand screening results using the MOE 2008.10 software (Force fields: AMBER99)

\begin{tabular}{c|l|c}
\hline S. No. & \multicolumn{1}{|c}{ Ligand } & $\Delta \mathrm{G}^{0}(\mathrm{kcal} / \mathrm{mol})$ \\
\hline 1 & Xanthosine_N3_TP_NS & -72.7155 \\
\hline 2 & Ribavirin_N2_N3_TP & -72.3952 \\
\hline 3 & Guanosine_S2_N3_DP_NS & -64.4642 \\
\hline 4 & Ribavirin_N2_S3_TP_NS & -63.7011 \\
\hline 5 & Xanthosine_N2_S3_DP & -63.4346 \\
\hline 6 & Inosine_N2_N3_TP & -63.3359 \\
\hline 7 & Viramidine_N3_DP & -61.521 \\
\hline 8 & Guanosine_N2_N3_TP_NS & -61.2829 \\
\hline 9 & Viramidine_N2_S3_TP_NS & -61.0656 \\
\hline 10 & Xanthosine_S2_N3_TP & -55.7377 \\
\hline 11 & Guanosine_N2_S3_TP_NS & -55.0802 \\
\hline 12 & Guanosine_S2_N3_TP & -54.6077 \\
\hline 13 & Deoxy_viramidine_N2_TP & -54.1252 \\
\hline 14 & Viramidine_N2_S3_DP & -53.8811 \\
\hline 15 & Ribavirin_N3_DP & -52.7499 \\
\hline 16 & Guanosine_N2_DP & -52.161 \\
\hline 17 & Viramidine_N3_TP & -52.1347 \\
\hline 18 & Viramidine_S2_N3_TP & -51.6483 \\
\hline 19 & Guanosine_N2_N3_DP & -51.5273 \\
\hline 20 & Xanthosine_S2_TP_NS & -51.1923 \\
\hline
\end{tabular}


RASĀYAN J. Chem.

Vol. 10 | No. 3 |910 - 921 | July - September | 2017

Table-2: Ligand screening results using the MOE 2008.10 software (Force fields: MMFF94x)

\begin{tabular}{c|l|c}
\hline S. No. & \multicolumn{1}{|c}{ Ligand } & $\Delta \mathrm{G}^{0}(\mathrm{kcal} / \mathrm{mol})$ \\
\hline 1 & Deoxy_Guanosien_N2_DP_NS & -47.2522 \\
\hline 2 & Ribaviri_N2_N3_P_NS & -41.1962 \\
\hline 3 & Guanosine_N2_N3_DP_NS & -37.9218 \\
\hline 4 & Emitricitabine_NH_TP & -31.1429 \\
\hline 5 & Deoxy_Ribavirin_TP_NS & -29.4037 \\
\hline 6 & Inosine_N2_N3_TP_NS & -28.9040 \\
\hline 7 & Inosine_N3_TP_NS & -26.1851 \\
\hline 8 & Viramidine_S2_S3_TP & -25.9986 \\
\hline 9 & Viramidine_S2_DP_NS & -23.9058 \\
\hline 10 & Inosine_N2_N3_DP_NS & -23.6565 \\
\hline 11 & Deoxy_Guanosine_TP & -22.4337 \\
\hline 12 & Guanosine_N3_DP_NS & -22.0661 \\
\hline 13 & Deoxy_Ribavirin_N2_TP & -21.3422 \\
\hline 14 & Inosine_S3_TP & -21.0119 \\
\hline 15 & Xanthosine_N2_DP & -20.6936 \\
\hline 16 & Guanosine_S3_DP_NS & -20.2306 \\
\hline 17 & Deoxy_Guanosine_N2_TP & -19.9055 \\
\hline 18 & Deoxy_Xanthosine_S2_TP & -19.6912 \\
\hline 19 & Deoxy_viramidine_N2_TP & -19.6111 \\
\hline 20 & Deoxy_Ribavirin_N2_DP_NS & -19.5728 \\
\hline & &
\end{tabular}

As we can see in these tables, the value of $\Delta \mathrm{G}^{0}(\mathrm{kcal} / \mathrm{mol})$ on MMFF94x produces higher value when compared with $\Delta \mathrm{G}^{0}$ on AMBER99 parameters. After completing the initial screening of 510 ligands, a total of 40 best ligand-screening results were forwarded from MOE 2008.10 for further docking simulation by using AutoDock Vina 1.0 software. The docking process was semi-oriented by using a flexible ligand and use the default number of rotatable bonds automatically by using AutoDock Tools, while the target enzyme for the docking process was made rigid. ${ }^{28}$ Docking results from AutoDock Vina 1.0 software were the best conformation of nine enzyme-ligand complexes with the chosen format. ${ }^{29}$ The results of docking simulation using AutoDock Tools, including the standards as well, can be seen in Table-3.

From the results in Table-3, it could be seen that the ribavirin triphosphate (RTP) has the lowest $\Delta \mathrm{G}_{\text {binding }}$ value at $6.3 \mathrm{kcal} / \mathrm{mol}$, much less than GTP and ribavirin (both at $5.2 \mathrm{kcal} / \mathrm{mol}$ ). Thus, it could be concluded that the NS5 Mtase would be more likely to bind to RTP rather with GTP or ribavirin. Out of several contact residues in the GTP, it was seen that there were four contact residues with amino acid residues of the NS5 Mtase. They were the active site of the DENV NS5 Mtase. ${ }^{30}$ Although ribavirin has the same affinity with GTP, it has better interaction than GTP with five residues, four of which was on the active site of DENV NS5 Mtase. The best interaction of the ligand was shown by the RTP which has eight contact residues with residues of NS5 Mtase, seven contact residues of which were on the active site of DENV NS5 Mtase. The resulted 40 new design ligands docking were screened based on binding energy and the contact residues. Thus, the ligand that considered to have better bond energy and the contact residues than standard ligands must meet the following criteria. The $\Delta \mathrm{G}_{\text {binding }}$ value must be lower than $-6.1 \mathrm{kcal} / \mathrm{mol}$. From the Table-3, it could be seen that 9 out of 40 ligands have lower $\Delta \mathrm{G}_{\text {binding }}$ value than GTP, RTP, and ribavirin, which means that they have higher binding affinity than the standards (except for Xanthosine_N2_S3_DP, which has 
RASĀYAN J. Chem.

Vol. 10 | No. 3 |910 - 921 | July - September | 2017

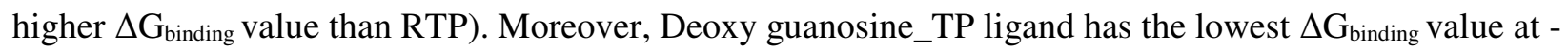
$6.9 \mathrm{kcal} / \mathrm{mol}$ and binds with seven residues of NS5 Mtase (Lys14, Lys22, Lys296, Ser150, Ser151, Ser214, and Thr215. The former five residues were located at the NS5 Mtase binding site).

Table-3: Results of ligand docking based on binding energy and the contact residues

\begin{tabular}{c|l|c|l}
\hline $\begin{array}{c}\text { S. } \\
\text { No. }\end{array}$ & \multicolumn{1}{|c}{ Ligand } & $\Delta \mathrm{G}(\mathrm{kcal} / \mathrm{mol})$ & \multicolumn{1}{c}{ Residues Contact } \\
\hline 1 & Deoxy guanosine_TP & -6.6 & Lys14; Lys22; Lys296; Ser150; Ser151; Ser214; Thr215 \\
\hline 2 & Guanosine_N2_DP & -6.8 & Asn18; Leu20; Gly21; Ser150; Ser151; Thr215 \\
\hline 3 & Deoxy ribavirin_N2_TP & -6.3 & Asn18; Leu20; Lys22; Lys29; Ser150; Ser151 \\
\hline 4 & Viramidine_S2_N3_TP & -6.3 & $\begin{array}{l}\text { Leu17; Leu20; Lys29; Ser150; Ser151; Lys181; Ser214; } \\
\text { Thr215; Glu217 }\end{array}$ \\
\hline 5 & Inosine_N2_N3_DP_NS & -6.4 & Lys14; Asn18; Leu20; Gly21; Lys22, Ser150 \\
\hline 6 & Inosine_N3_TP_NS & -6.3 & Lys14; Leu17; Leu20; Ser150; Ser151; Ser214; Thr215 \\
\hline 7 & Xanthosine_S2_TP_NS & -6.3 & Lys14; Asn18; Leu20; Lys22; Lys29; Ser150; Ser151 \\
\hline 8 & Xanthosine_N2_S3_DP & -6.1 & Lys14; Asn18; Leu20; Lys29; Ser150; Ser151 \\
\hline 9 & Xanthosine_N3_TP_NS & -6.4 & Leu17; Asn18; Leu20; Lys22; Ser151 \\
\hline S1 & GTP & -5.2 & Lys14; Lys29; Ser150, Ser151 \\
\hline S2 & Ribavirin & -5.2 & Lys14; Leu17; Asn18; Ser150; Glu157 \\
\hline S3 & RTP & -6.3 & $\begin{array}{l}\text { Lys14; Asn18; Lue20; Gly21; Lys22; Lys 29; Ser150; } \\
\text { Ser151 }\end{array}$ \\
\hline
\end{tabular}

From the Figures-3a, 3b, and 3c above, the interaction of the ligand to the surface could be seen from the cavity of the catalytic site (active site) of DENV NS5 Mtase. It could be seen whether the structure of the ligand was appropriate to form a complex with the active DENV NS5 Mtase.

Viramidine_S2_N3_TP Ligand binds with Leu17, Leu20, Phe25, Lys29, Ser150, and Ser151. In Fig.-4, it is shown that the aromatic group and a phosphate group interact with the amino acid residues of the active site of the DENV NS5 Mtase. The active sites have a group of aromatic Phe25 and Lys29 with the phosphate group.

Xanthosine_N2_S3_DP Ligand interactions with the DENV NS5 Mtase above showed some of the interactions between amino acid residues (Fig.-5). It occurred at the active site of DENV NS5 Mtase with through hydrogen bonds, and bonds, among others, with Lys14, Asn18, Leu20, Lys29, Ser150, and Ser151. In Fig.-5, it shows that the phosphate group interacts with the amino acid residues of the DENV NS5 Mtase active site.

Ligand interactions Inosin_N2_N3_DP_NS with DENV NS5 Mtase above shows some of the interactions between amino acid residues at the active site of Mtase with ligand through hydrogen bonding (Fig.-6). It occurred with Lys14, Asn18, Leu20, Lys22, and Ser150. In Fig.-7, it was shown that the modified functional group and N'3 N'2 sugar ribose of inosine interact with Asn18, one of the amino acid residues of the active site of the DENV NS5 Mtase. Moreover, the modified non-cyclic carbonyl group gave a fairly good interaction with Lys14.

\section{Bioavailability, Toxicity, and Synthetic Accessibility Prediction}

Out of the nine ligands that were selected based on the docking analysis (affinity and interaction), we only chose several ligands that have good drug properties, based on their oral bioavailability prediction.

The oral bioavailability prediction of the best ligands can be done by referring to the Lipinski's Rule of Five $^{31,32}$, which was done by using the online software provided by SCFBIO-IITD (http://www.scfbioiitd.res.in/software/drugdesign/lipinski.jsp) ${ }^{33}$. The results of this screening are shown in Table-4. 
RASĀYAN $J$. Chem.

Vol. 10 | No. 3 |910 - 921 | July - September | 2017

Based on test results Lipinski's Rule of Five, all of the ligands were qualified, because the minimum criteria of this test were to meet within two or more of five conditions Lipinski's Rule.

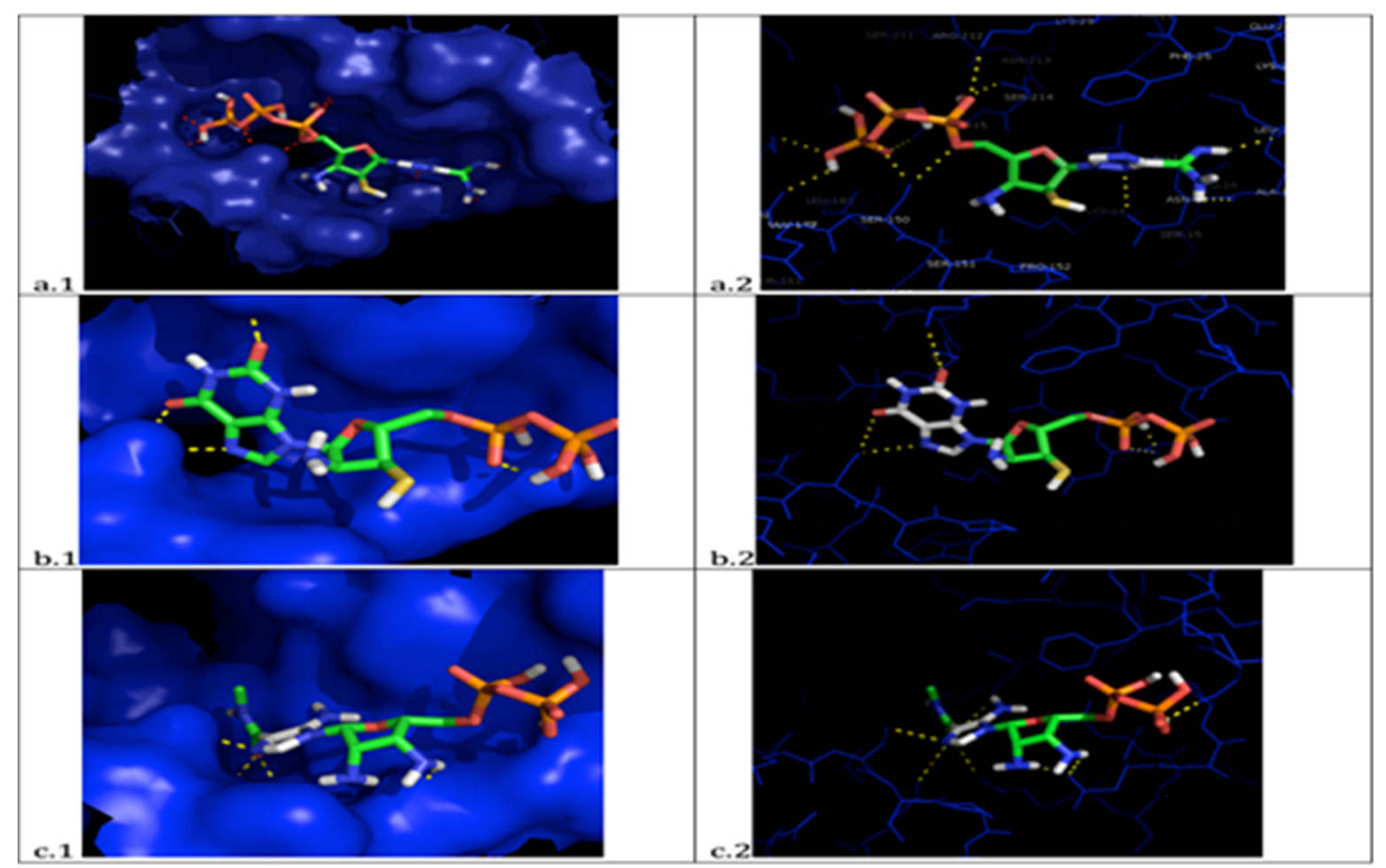

Fig.-3: (a) 3D Viramidine_S2_N3_TP Ligand Interaction, (b) 3D Xanthosine_N2_S3_DP Ligand Interaction, (c) 3D Inosin_N2_N3_DP_NS Ligand Interaction with DENV NS5 Mtase.

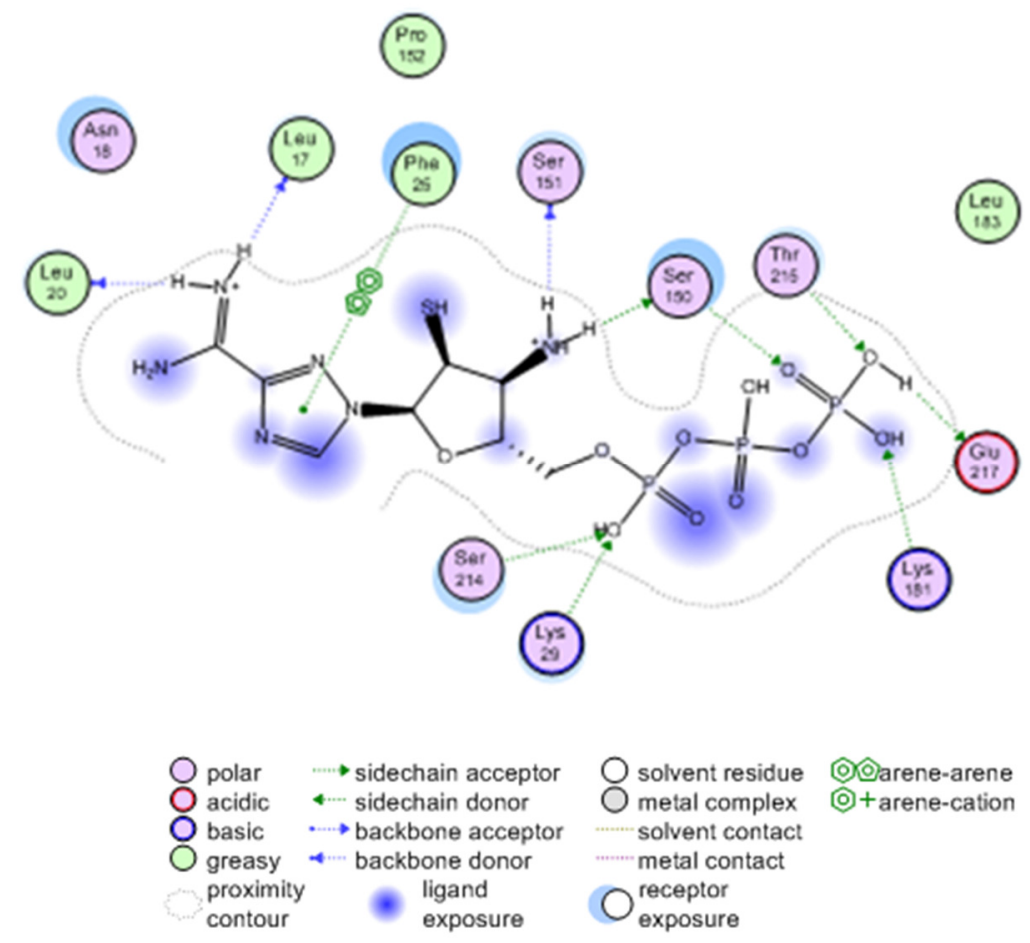

Fig.-4: The 2D visualization of Viramidine_S2_N3_TP ligand interaction with NS5 methyltransferase dengue virus using the MOE 2008.10 software. 
RASĀYAN J. Chem.

Vol. 10 | No. 3 |910 - 921 | July - September | 2017

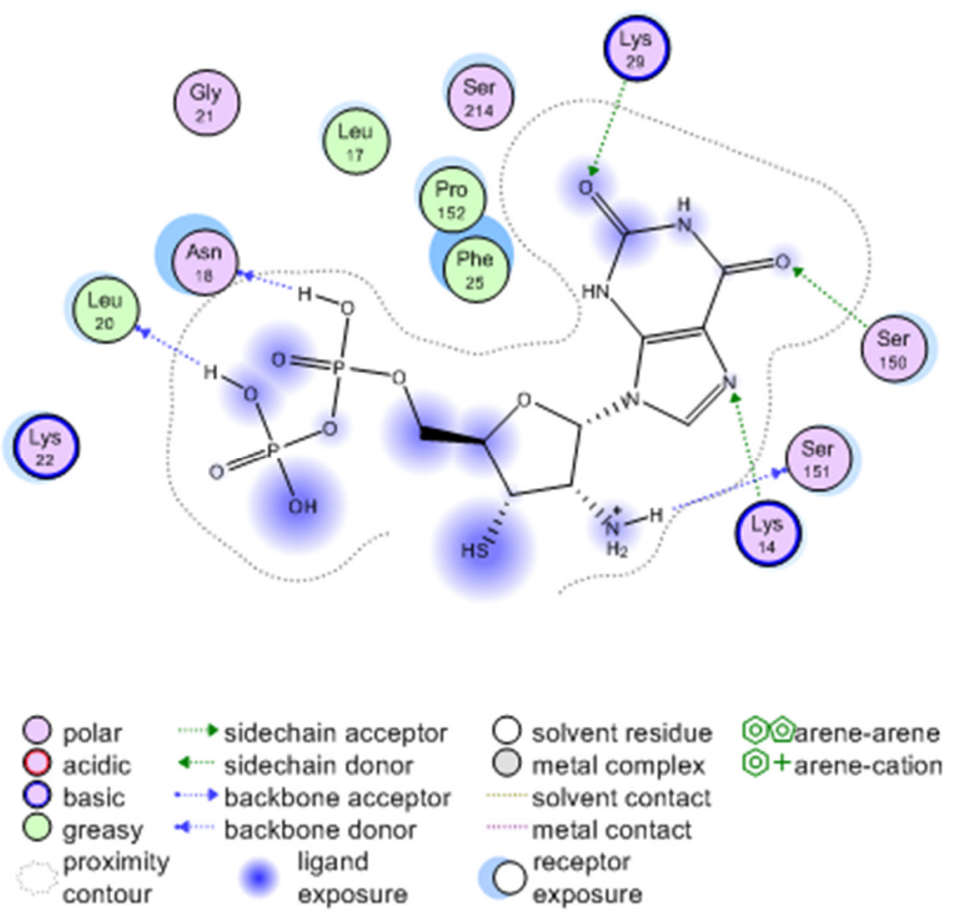

Fig.-5: The 2D visualization of Xanthosine_N2_S3_DP ligand interaction with NS5 methyltransferase dengue virus using the MOE 2008.10 software.

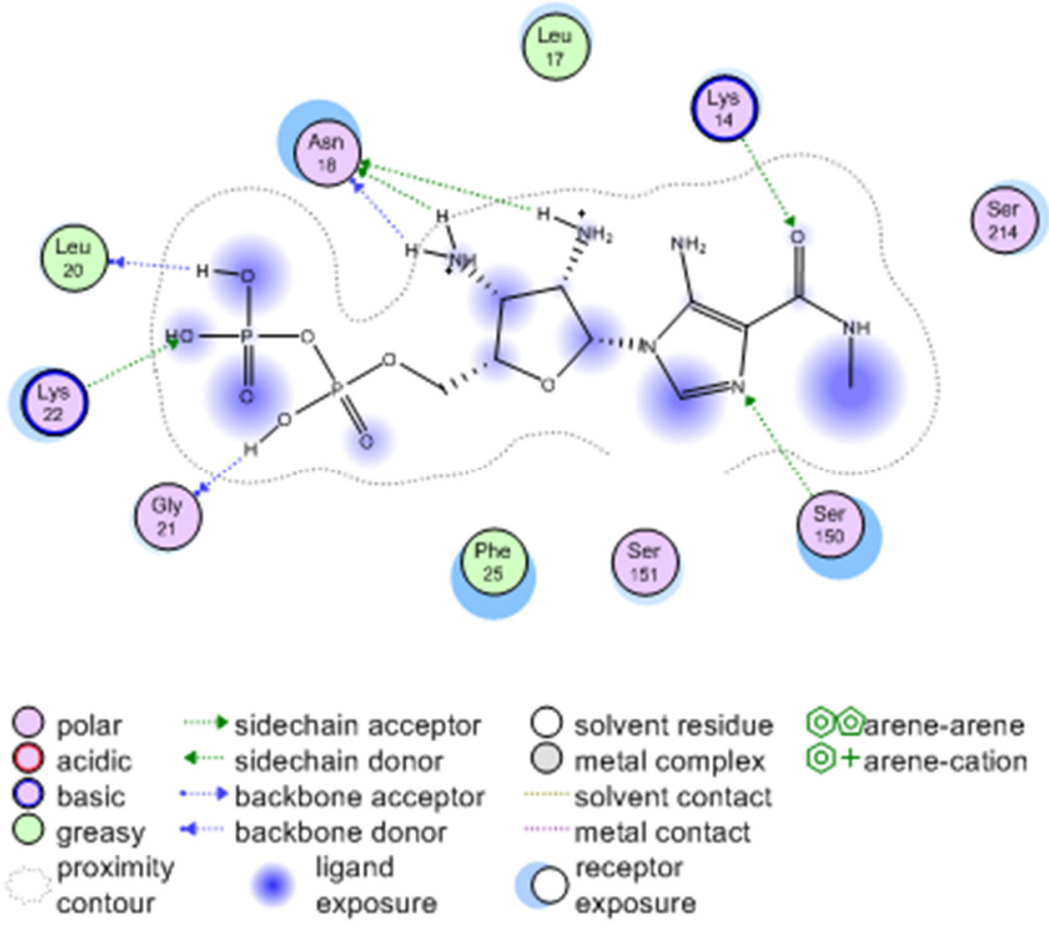

Fig.-6: The 2D visualization of Inosin_N2_N3_DP_NS ligand Interaction with NS5 methyltransferase dengue virus using the MOE 2008.10 software.

The screening was done with the ligand properties using online software Molinspirations (www.molinspirations.com) to determine some properties of the ligand such as high total polar surface area (TPSA) value, $\log$ P, and noRTB (number of rotatable bonds). 


\section{RASĀYAN J. Chem.}

Vol. 10 | No. 3 |910 - 921 | July - September | 2017

Table-5 and 6 show the results of the screening of oral bioavailability standards and ligand. Oral bioavailability screening results show that nothing met all the criteria based on Veber's Rules and Egan's Rules. However, RTP did not pass the Veber's and Egan's Rules due to the abundance of hydrogen bond donors and TPSA value, respectively. Moreover, the GTP and ribavirin have almost the same problem due to its molecular weight, hydrogen bond donors/acceptors, and TPSA value. Based on the results of screening (Lipinski's Rule of Five and screening for oral bioavailability) the design of ligands that have high levels of drug-likeness is Viramidine_S2_N3_TP (based on log P value), and Inosin_N2_N3_DP_NS Xanthosine_N2_S3_DP.

Table-4: The results of ligand screening based on drug-likeness

\begin{tabular}{c|l|c|c|c|c|c}
\hline S. No. & \multicolumn{1}{|c}{ Ligand } & $\begin{array}{c}\text { Molecular } \\
\text { weight } \\
\text { (Dalton) }\end{array}$ & $\begin{array}{c}\text { H-bond } \\
\text { donor }\end{array}$ & $\begin{array}{c}\text { H-bond } \\
\text { acceptor }\end{array}$ & logP & $\begin{array}{c}\text { Molar } \\
\text { Refractivity }\end{array}$ \\
\hline 1 & GTP & 525 & 9 & 12 & -3.577 & 96.336 \\
\hline 2 & Ribavirin & 244 & 5 & 7 & -5.066 & 44.749 \\
\hline 3 & RTP & 484 & 8 & 10 & -2.512 & 75.796 \\
\hline 4 & Deoxy guanosine_TP & 509 & 8 & 11 & -1.516 & 95.062 \\
\hline 5 & Guanosine_N2_DP & 444 & 8 & 10 & nd & nd \\
\hline 6 & Deoxy ribavirin_N2_TP & 467 & 8 & 9 & 0.552 & 76.879 \\
\hline 7 & Viramidine_S2_N3_TP & 498 & 9 & 10 & 1.390 & 64.532 \\
\hline 8 & Inosine_N2_N3_DP_NS & 430 & 10 & 9 & nd & nd \\
\hline 9 & Inosine_N3_TP_NS & 511 & 10 & 11 & -1.058 & 61.081 \\
\hline 10 & Xanthosine_S2_TP_NS & 542 & 8 & 10 & -0.479 & 63.482 \\
\hline 11 & Xanthosine_N2_S3_DP & 459 & 7 & 9 & 0.323 & 53.080 \\
\hline 12 & Xanthosine_N3_TP_NS & 525 & 10 & 11 & -1.831 & 58.967 \\
\hline
\end{tabular}

Table-5: Oral bioavailability screening results of the standards and ligand, based on Veber's Rule

\begin{tabular}{c|l|c|c|c}
\hline S. No. & \multicolumn{1}{|c|}{ Ligand } & $\begin{array}{c}\text { H-bond acceptor } \\
\text { + H-bond donor }\end{array}$ & TPSA ( $)$ & rotatable bond \\
\hline 1 & GTP* & 21 & 292.604 & 8 \\
\hline 2 & Ribavirin* & 12 & 143.730 & 3 \\
\hline 3 & RTP* & 18 & 283.329 & 9 \\
\hline 4 & Deoxy guanosine_TP & 19 & 272.376 & 8 \\
\hline 5 & Guanosine_N2_DP & 18 & 251.866 & 6 \\
\hline 6 & Deoxy ribavirin_N2_TP & 17 & 268.896 & 9 \\
\hline 7 & Viramidine_S2_N3_TP & 19 & 275.677 & 9 \\
\hline 8 & Inosine_N2_N3_DP_NS & 19 & 247.521 & 7 \\
\hline 9 & Inosine_N3_TP_NS & 21 & 288.259 & 9 \\
\hline 10 & Xanthosine_S2_TP_NS & 18 & 279.307 & 9 \\
\hline 11 & Xanthosine_N2_S3_DP & 16 & 232.101 & 6 \\
\hline 12 & Xanthosine_N3_TP_NS & 21 & 305.330 & 9 \\
\hline
\end{tabular}

Not only the oral bioavailability prediction, but the toxicity prediction is also helpful to find a ligand that has good drug-like properties. In this research, we used Toxtree software to determine the toxicity properties of our ligands. The results of this prediction test are shown in Table-7. From the results of toxicity prediction using Toxtree, it looks that that ligand has no genotoxic and non-genotoxic SAS (Table-7). The QSAR 
results show that they are not mutagenic and carcinogens. Based on CAESA, Viramidine_S2_N3_TP and Inosine_N2_N3_DP_NS synthesis were predicted through tetrahydrofuran compounds, whereas the synthesis of Xanthosine_N2_S3_DP ligand was predicted through urea compounds. Different things might have happened if their synthesis started from the original compound. To obtain more representative data, it is necessary to know the SA value of the basic compounds of the standards and the three ligands. CAESA results for the basic compounds from RTP and ligand compounds are shown in Table-8.

Table-6: Oral bioavailability screening results of the standards and ligand, based on Egan's Rule

\begin{tabular}{c|l|c|c}
\hline S. No. & \multicolumn{1}{|c|}{ Ligand } & $\log \mathrm{P}$ & TPSA (A) \\
\hline 1 & GTP* & -3.577 & 292.604 \\
\hline 2 & Ribavirin* & -5.066 & 143.730 \\
\hline 3 & RTP* & -2.512 & 283.329 \\
\hline 4 & Deoxy guanosine_TP & -1.516 & 272.376 \\
\hline 5 & Guanosine_N2_DP & nd & 251.866 \\
\hline 6 & Deoxy ribavirin_N2_TP & 0.552 & 268.896 \\
\hline 7 & Viramidine_S2_N3_TP & 1.390 & 275.677 \\
\hline 8 & Inosine_N2_N3_DP_NS & nd & 247.521 \\
\hline 9 & Inosine_N3_TP_NS & -1.058 & 288.259 \\
\hline 10 & Xanthosine_S2_TP_NS & -0.479 & 279.307 \\
\hline 11 & Xanthosine_N2_S3_DP & 0.323 & 232.101 \\
\hline 12 & Xanthosine_N3_TP_NS & -1.831 & 305.330 \\
\hline
\end{tabular}

Table-7: Toxicity Prediction Results in Toxtree

\begin{tabular}{l|l|l|l}
\hline \multicolumn{1}{c|}{ Toxicity Prediction } & $\begin{array}{l}\text { Viramidine_S2_N3 _TP } \\
\text { _TP }\end{array}$ & $\begin{array}{l}\text { Inosin_N2_N3_D } \\
\text { P_NS }\end{array}$ & $\begin{array}{l}\text { Xanthosine_N2_S } \\
\text { 3_DP }\end{array}$ \\
\hline $\begin{array}{l}\text { Structural Alert for nongenotoxic } \\
\text { carcinogenicity }\end{array}$ & No & No & No \\
\hline Structural Alert for genotoxic carcinogenicity & No & No & No \\
\hline Potential carcinogen based on QSAR & No & No & No \\
\hline $\begin{array}{l}\text { Potential S. Typhimurium TA100 mutagen } \\
\text { based on QSAR }\end{array}$ & No & No & No \\
\hline Negative for genotoxic carcinogenicity & Yes & Yes & Yes \\
\hline Negative for nongenotoxic carcinogenicity & Yes & Yes & Yes \\
\hline
\end{tabular}

Table-8: Predicted results for the SA standard precursors and ligands

\begin{tabular}{c|l|l|l|l}
\hline S. No. & Ligand & $\begin{array}{l}\text { Structural } \\
\text { Complexity }\end{array}$ & Starting Materials & $\begin{array}{l}\text { Synthetic } \\
\text { Accessibility }\end{array}$ \\
\hline 1 & Ribavirin & $0 \%$ & $100 \%$ & $100 \%$ \\
\hline 2 & Viramidine & $49 \%$ & $31 \%$ & $46 \%$ \\
\hline 3 & Inosin & $0 \%$ & $100 \%$ & $100 \%$ \\
\hline 4 & Xanthosine & $0 \%$ & $100 \%$ & $100 \%$ \\
\hline
\end{tabular}

As for the possibility of viramidine compound synthesis, the process is much more complicated with the synthesis ability of $46 \%$. Although viramidine, which is a basic compound of Viramidine_S2_N3_TP ligand, do not give a 100\% value, Viramidine_S2_N3_TP is still possible to be synthesized by the wet lab. 
Overall, based on data obtained from the CAESA, those ligands could be synthesized by a wet lab in addition to RTP.

\section{CONCLUSION}

In this research, it was found that the three best candidate ligand compounds are Viramidine_S2_N3_TP, Inosine_N2_N3_DP_NS, and Xanthosine_N2_S3_DP. They have a good binding affinity value docking results, which is $-6.3 \mathrm{kcal} / \mathrm{mol},-6.4 \mathrm{kcal} / \mathrm{mol}$, and $-6.1 \mathrm{kcal} / \mathrm{mol}$ respectively. Similarly, interactions of Inosine_N2_N3_DP_NS and Xanthosine_N2_S3_DP with important residues of the active site of the DENV NS5 Mtase gave good results on a drug test likeness. They met three out of five Lipinski's Rule. The Viramidine_S2_N3_TP ligand was chosen because it has a good $\log$ P value, which is 1.39. Predicted results for synthetic accessibility (SA) of the three ligands showed good results compared to the existing standard RTP drug. Inosine_N2_N3_DP_NS and Xanthosine_N2_S3_DP ligands have predictive value of 43\% SA, Viramidine_S2_N3_TP SA ligand gives the predicted value by $44 \%$, while the RTP as a standard SA, has the predictive value $41 \%$. Therefore, the molecular dynamics simulation of these compounds can be performed to determine their stability against DENV NS5 Mtase before this study can be validated later in the wet experiment.

\section{ACKNOWLEDGEMENT}

This research was supported by Hibah Riset Unggulan Universitas Indonesia No: 1325/UN2.R12/PPM.00.00/2015 and Hibah Penelitian Utama Perguruan Tinggi 2017 No: 3162/UN2.R3.1/PPM.00.01/2017. The authors are grateful to Directorate of Research and Community Engagement (DRPM), Universitas Indonesia for their support toward this research. USFT and DK were supervising this research and creating the general pipeline of this research, FW was working on technical assistance, while AAP and MAFN were preparing the English manuscript, and re-verified the whole experiment. The authors declared that there is no competing interest in this research.

\section{REFERENCES}

1. T.P. Monath, Proc. Natl. Acad. Sci. U. S. A. 91, 2395 (1994).

2. J.R. Stephenson, Bull. World Health Organ. 83, 308 (2005).

3. S.B. Halstead, in Annu. Rev. Entomol. (2008), pp. 273-291.

4. J.P. Messina, O.J. Brady, T.W. Scott, C. Zou, D.M. Pigott, K.A. Duda, S. Bhatt, L. Katzelnick, R.E. Howes, K.E. Battle, C.P. Simmons, and S.I. Hay, Trends Microbiol. 22, 138 (2014).

5. M.S. Mustafa, V. Rasotgi, S. Jain, and V. Gupta, Med. J. Armed Forces India 71, 67 (2015).

6. W. Chen, H. Kawano, R. Men, D. Clark, and C.J. Lai, J. Virol. 69, 5186 (1995).

7. M.M.F. Alen and D. Schols, J. Trop. Med. 2012, (2012).

8. M.G. Guzman, D.J. Gubler, A. Izquierdo, E. Martinez, and S.B. Halstead, Nat. Rev. Dis. Prim. 2, 16055 (2016).

9. C.G. Noble and P.Y. Shi, Antiviral Res. 96, 115 (2012).

10. S.P. Lim, C.G. Noble, and P.-Y. Shi, Antiviral Res. 119, 57 (2015).

11. D. Benarroch, M.-P. Egloff, L. Mulard, C. Guerreiro, J.-L. Romette, and B. Canard, J. Biol. Chem. 279, 35638 (2004).

12. J. Li, S.P. Lim, D. Beer, V. Patel, D. Wen, C. Tumanut, D.C. Tully, J.A. Williams, J. Jiricek, $\quad$ J.P. Priestle, J.L. Harris, and S.G. Vasudevan, J. Biol. Chem. 280, 28766 (2005).

13. S. Idrus, U. Tambunan, and A.A. Zubaidi, Bioinformation 8, 348 (2012).

14. U.S.F. Tambunan, H. Zahroh, B.B. Utomo, and A.A. Parikesit, Bioinformation 10, 23 (2014).

15. V.K. Morya, S. Kumari, and E. Kim, Clin. Proteomics 9, 1 (2012).

16. P. Ertl and A. Schuffenhauer, J. Cheminform. 1, 8 (2009).

17. P.R. Kakadiya, T.G. Chandrashekhar, S. Ganguly, D.K. Singh, and V. Singh, Anal. Chem. Insights 6, 21 (2011).

18. C. Bagshaw, J. Cell Sci. 114, 459 (2001).

19. C.A. Motti, D.G. Bourne, J.N. Burnell, J.R. Doyle, D.S. Haines, C.H. Liptrot, L.E. Llewellyn, S. Ludke, A. Muirhead, and D.M. Tapiolas, Appl. Environ. Microbiol. 73, 1921 (2007). 
20. B.J. Geiss, A.A. Thompson, A.J. Andrews, R.L. Sons, H.H. Gari, S.M. Keenan, and O.B. Peersen, J. Mol. Biol. 385, 1643 (2009).

21. W. James, Curr. Opin. Pharmacol. 1, 540 (2001).

22. D.L. Wheeler, C. Chappey, A.E. Lash, D.D. Leipe, T.L. Madden, G.D. Schuler, T.A. Tatusova, and B.A. Rapp, Nucleic Acids Res. 44, D7 (2016).

23. P. Benkert, M. Biasini, and T. Schwede, Bioinformatics 27, 343 (2011).

24. M. Biasini, S. Bienert, A. Waterhouse, K. Arnold, G. Studer, T. Schmidt, F. Kiefer, T.G. Cassarino, M. Bertoni, L. Bordoli, and T. Schwede, Nucleic Acids Res. 42, W252 (2014).

25. M.-P.P. Egloff, E. Decroly, H. Malet, B. Selisko, D. Benarroch, F. Ferron, and B. Canard, J. Mol. Biol. 372, 723 (2007).

26. U.S.F. Tambunan and S. Alamudi, Bioinformation 5, 250 (2010).

27. M. Podvinec, S.P. Lim, T. Schmidt, M. Scarsi, D. Wen, L.S. Sonntag, P. Sanschagrin, P.S. Shenkin, and T. Schwede, J. Med. Chem. 53, 1483 (2010).

28. U.S.F. Tambunan, R. Bakri, T. Prasetia, A.A. Parikesit, and D. Kerami, Bioinformation 9, 696 (2013).

29. V.Y. Tanchuk, V.O. Tanin, and A.I. Vovk, Chem. Biol. Drug Des. 80, 121 (2012).

30. H. Kroschewski, S.P. Lim, R.E. Butcher, T.L. Yap, J. Lescar, P.J. Wright, S.G. Vasudevan, and A.D. Davidson, J. Biol. Chem. 283, 19410 (2008).

31. R. Bakri, A.A. Parikesit, C.P. Satriyanto, D. Kerami, and U.S.F. Tambunan, Adv. Bioinformatics 7, 1 (2014).

32. C.A. Lipinski, F. Lombardo, B.W. Dominy, and P.J. Feeney, Adv. Drug Deliv. Rev. 23, 3 (1997).

33. B. Jayaram, T. Singh, G. Mukherjee, A. Mathur, S. Shekhar, and V. Shekhar, BMC Bioinformatics 13 Suppl 1, S7 (2012).

[RJC-1613/2017] 